\title{
Electron-boson-interaction induced particle-hole symmetry breaking of conductance into subgap states in superconductors
}

\author{
F. Setiawan $\odot^{1,2,{ }^{*}}$ and Jay D. Sau ${ }^{2}$ \\ ${ }^{1}$ Pritzker School of Molecular Engineering, University of Chicago, 5640 South Ellis Avenue, Chicago, Illinois 60637, USA \\ ${ }^{2}$ Condensed Matter Theory Center and Joint Quantum Institute, Department of Physics, \\ University of Maryland, College Park, Maryland 20742, USA
}

(Received 6 August 2020; revised 19 July 2021; accepted 23 July 2021; published 11 August 2021)

\begin{abstract}
Particle-hole symmetry (PHS) of conductance into subgap states in superconductors is a fundamental consequence of a noninteracting mean-field theory of superconductivity. The breaking of this PHS has been attributed to a noninteracting mechanism, i.e., quasiparticle poisoning (QP), a process detrimental to the coherence of superconductor-based qubits. Here we show that the ubiquitous electron-boson interactions in superconductors can also break the PHS of subgap conductances. We study the effect of such couplings on the PHS of subgap conductances in superconductors using both the rate equation and Keldysh formalism, which have different regimes of validity. In both regimes, we found that such couplings give rise to a particle-hole asymmetry in subgap conductances which increases with increasing coupling strength, increasing subgap-state particle-hole content imbalance and decreasing temperature. Our proposed mechanism is general and applies even for experiments where the subgap-conductance PHS breaking cannot be attributed to QP.
\end{abstract}

DOI: 10.1103/PhysRevResearch.3.L032038

\section{INTRODUCTION}

Subgap states in superconductors are key features of topological superconducting phases [1-13] which offer great promise for quantum information processing [14,15]. Tunneling transport into such Andreev bound states (ABSs) provides the most direct and commonly employed method to detect them [16-19]. (Hereafter ABS refers to any subgap state in superconductors.) Most of our understanding of tunneling into superconductors is based on the celebrated Blonder-Tinkham-Klapwijk (BTK) formalism [20]. One universal consequence of this theory is a precise particle-hole symmetry (PHS) of the conductance into any ABS in a superconductor $[18,21,22]$. Specifically, this theory predicts that the differential conductance at a positive voltage $V$ inside the superconducting gap precisely match its counterpart value at $-V$. This symmetry has been shown to be a consequence of the PHS of the mean-field Hamiltonian used in the BTK formalism. However, numerous experiments over two decades [3-8,11,23-34] have often observed particle-hole (PH) asymmetric subgap conductances. One way to reconcile this $\mathrm{PH}$ asymmetry with the BTK theory is to introduce quasiparticle poisoning induced either by coupling the ABS to a fermionic bath $[22,35,36]$ or through a relaxation process from the ABS to the superconductor's quasiparticle continuum [37].

\footnotetext{
*setiawan@uchicago.edu

Published by the American Physical Society under the terms of the Creative Commons Attribution 4.0 International license. Further distribution of this work must maintain attribution to the author(s) and the published article's title, journal citation, and DOI.
}

Quasiparticle poisoning (QP) [38-40] refers to a process where an electron tunnels from the bulk of the superconductor to an ABS which changes the occupation (parity) of the ABS. Since the parity is used as the qubit state, QP then introduces bit-flip errors [41-43]. Moreover, as QP breaks the PHS of subgap conductances $[22,35,36]$, one may be tempted to associate the PH asymmetry to short qubit lifetime. We will show that this correlation is not true in general, as contrary to commonly held belief, the PH asymmetry can also arise without QP.

In this paper, we propose a generic mechanism for PHS breaking of subgap conductances without changing the superconductor's parity state, namely, the coupling between ABSs and bosonic modes. While quantum tunneling in dissipative systems has been widely studied $[44,45]$, previous works consider coupling between bosonic baths and superconductors without ABSs. Motivated by tunneling experiments into ABSs [3-8,11,23-34], here we study tunneling transport from a normal lead into an ABS coupled to bosonic modes, e.g., phonons [46,47], plasmons [48], or electromagnetic fields [49], in the superconductor. Our system has a local fermion parity analogous to the spin-boson model [50] with a caveat that our ABSs can participate in transport. Crucially, our study of transport into an ABS coupled to bosonic modes and its relation to PHS breaking of subgap conductances has not been undertaken before. To this end, we present ways to enforce fermion-parity conservation in treating interaction effects on transport into ABSs. We consider two different limits: weak and strong tunneling regimes where the ABS-lead tunnel strength is smaller and larger than the thermal broadening $\sim k_{B} T$, respectively. The weak tunneling limit is studied using the rate equation [51,52], which is valid for all values of ABS-boson coupling strength where the tunneling rates are calculated using 


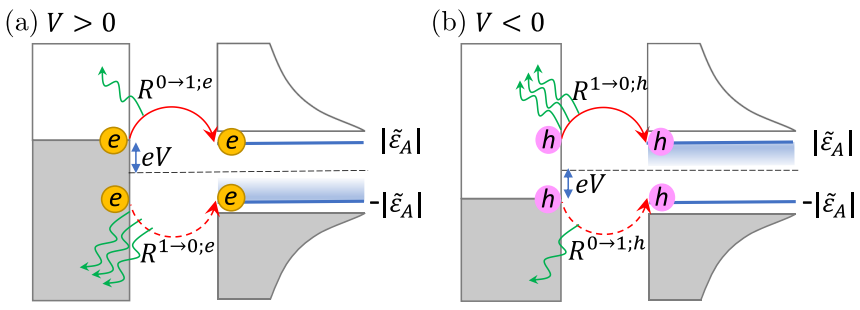

FIG. 1. Sequential tunneling from the lead (grey rectangles) to the ABS (blue lines). Tunneling of (a) electrons and (b) holes give rise to current at (a) positive and (b) negative voltages, respectively [Eq. (1)]. The first tunneling flips the ABS occupancy $n$ from $0 \rightarrow 1$ and occurs with rates (a) $R^{0 \rightarrow 1 ; e}$ or (b) $R^{0 \rightarrow 1 ; h}$. The second tunneling, which flips $n$ from $1 \rightarrow 0$, occurs with rates (a) $R^{1 \rightarrow 0 ; e}$ or (b) $R^{1 \rightarrow 0 ; h}$. Without bosonic baths, $R^{0 \rightarrow 1 ; e}=R^{1 \rightarrow 0 ; h}$ and $R^{1 \rightarrow 0 ; e}=R^{0 \rightarrow 1 ; h}$ giving a $\mathrm{PH}$ symmetric conductance. However, in the presence of bosonic baths, the second tunneling occurs with a higher rate since it can transfer lead electrons within a larger energy range near the ABS energy (shaded blue region) where the energy difference can be dumped by emitting bosons (green squiggly lines). Therefore, $R^{0 \rightarrow 1 ; e} \neq R^{1 \rightarrow 0 ; h}$ and $R^{1 \rightarrow 0 ; e} \neq R^{0 \rightarrow 1 ; h}$ resulting in a $\mathrm{PH}$ asymmetric conductance.

Fermi's Golden Rule (FGR). In the strong tunneling limit, we study the transport using the Keldysh formalism and treat the ABS-boson coupling within the mean-field approximation.

\section{PARTICLE-HOLE SYMMETRY/ASYMMETRY FROM FERMI'S GOLDEN RULE}

We begin by using FGR to show that while subgap conductances in gapped superconductors (superconductors without baths) preserve PHS even with interactions (including strongly correlated superconductors), the PHS is broken for superconductors with gapless excitations (e.g., phonons, quasiparticles, etc.). The simplest application of FGR $[23,53,54]$ considers the conductance into an ABS at positive [Fig. 1(a)] and negative subgap energies [Fig. 1(b)] to arise from the tunneling of electrons and holes, respectively, into the ABS (changing the ABS occupancy $n$ from $0 \rightarrow 1$ ). The tunneling rates of electrons $\left[R^{0 \rightarrow 1 ; e}\right.$ in Fig. 1(a)] and holes $\left[R^{0 \rightarrow 1 ; h}\right.$ in Fig. 1(b)] can be calculated from FGR to be proportional to the particle and hole component of the ABS wave function, respectively. This suggests that the tunneling conductance into an ABS with different weights of particle and hole component is $\mathrm{PH}$ asymmetric. However, this simple argument implicitly assumes the presence of QP [22], which empties out the electron from the ABS after each tunneling event such that its occupancy $n$ returns to $n=0$. This implicit assumption can be avoided by taking into account the change in the ABS occupancy $n=0,1$ after each tunneling.

As seen in Fig. 1(a), the electron tunneling flips $n$ either from $0 \rightarrow 1$ (with a rate $R^{0 \rightarrow 1 ; e}$ ) or vice versa (with a rate $R^{1 \rightarrow 0 ; e}$ ). Since each tunneling event flips $n \rightarrow \bar{n} \equiv 1-n$, a full cycle of transferring a pair of electrons returns the occupancy to the initial $|n=0\rangle$ occupancy state. The total time for this process that transfers a charge of $2 e$ is $\tau=$ $\left(R^{0 \rightarrow 1 ; e}\right)^{-1}+\left(R^{1 \rightarrow 0 ; e}\right)^{-1}$ leading to a current $I=2 e / \tau$. Combining this result with the analogous argument for negative voltages [Fig. 1(b)] leads to the expression for the tunneling current (we give a more detailed derivation later):

$$
I= \begin{cases}2 e \frac{R^{0 \rightarrow 1 ; e} R^{1 \rightarrow 0 ; e}}{R^{0 \rightarrow 1 ; e}+R^{1 \rightarrow 0 ; e}} & \text { for } e V \gtrsim\left|\tilde{\varepsilon}_{\mathrm{A}}\right|+k_{\mathrm{B}} T, \\ -2 e \frac{R^{0 \rightarrow 1 ; h} R^{1 \rightarrow 0 ; h}}{R^{0 \rightarrow 1 ; h}+R^{1 \rightarrow 0 ; h}} & \text { for } e V \lesssim-\left(\left|\tilde{\varepsilon}_{\mathrm{A}}\right|+k_{\mathrm{B}} T\right),\end{cases}
$$

where $\tilde{\varepsilon}_{\mathrm{A}}$ is the interaction-renormalized ABS energy, $k_{\mathrm{B}}$ is the Boltzmann constant, and $T$ is the temperature. The constraints on the voltage $V$ in Eq. (1) are needed to separate the electron and hole tunneling shown in Fig. 1. Using FGR, we calculate the electron and hole tunneling rates as $R^{n \rightarrow \bar{n} ; e} \propto\left|\left\langle\bar{n}\left|\hat{d}_{\mathrm{A}}^{\dagger}\right| n\right\rangle\right|^{2}$ and $R^{n \rightarrow \bar{n} ; h} \propto\left|\left\langle\bar{n}\left|\hat{d}_{\mathrm{A}}\right| n\right\rangle\right|^{2}$, where $\hat{d}_{\mathrm{A}}^{\dagger}$ and $\hat{d}_{\mathrm{A}}$ are the electron and hole creation operators in the ABS, respectively. Since $R^{0 \rightarrow 1 ; e}=R^{1 \rightarrow 0 ; h}$ and $R^{1 \rightarrow 0 ; e}=R^{0 \rightarrow 1 ; h}$, the current [Eq. (1)] is antisymmetric $I(V)=-I(-V)$ and the corresponding subgap conductance shows PHS for a gapped superconductor even with interactions (including strongly correlated superconductors). However, as shown below, this PHS is broken in the presence of bosonic baths.

\section{MODEL I. TUNNELING INTO BOSON-COUPLED ABS}

We consider tunneling of electrons or holes from a onedimensional normal lead into an ABS coupled to bosonic modes (e.g., phonons); see Fig. 1. The total Hamiltonian comprises the Hamiltonian of a boson-coupled ABS, lead, and tunnel coupling, i.e, $\hat{H}=\hat{H}_{\mathrm{A}}+\hat{H}_{\mathrm{L}}+\hat{H}_{\mathrm{T}}$, where

$$
\begin{aligned}
\hat{H}_{\mathrm{A}} & =\varepsilon_{\mathrm{A}} \hat{\gamma}^{\dagger} \hat{\gamma}+\lambda \hat{\gamma}^{\dagger} \hat{\gamma}\left(\hat{b}^{\dagger}+\hat{b}\right)+\Omega \hat{b}^{\dagger} \hat{b}, \\
\hat{H}_{\mathrm{L}} & =\sum_{k} \varepsilon_{\mathrm{L}, k} \hat{c}_{\mathrm{L}, k}^{\dagger} \hat{c}_{\mathrm{L}, k}, \\
\hat{H}_{\mathrm{T}} & =t \hat{c}_{\mathrm{L}}^{\dagger} \hat{d}_{\mathrm{A}}+\text { H.c. }
\end{aligned}
$$

Here $\varepsilon_{\mathrm{A}}$ is the ABS energy, $\hat{\gamma}\left(\hat{\gamma}^{\dagger}\right)$ is the Bogoliubov annihilation (creation) operator of the ABS, $\lambda$ is the ABS-boson coupling strength, $\hat{b}\left(\hat{b}^{\dagger}\right)$ is the boson annihilation (creation) operator, and $\Omega$ is the boson frequency. The operator $\hat{c}_{\mathrm{L}, k}$ $\left(\hat{c}_{\mathrm{L}, k}^{\dagger}\right)$ annihilates (creates) the lead electron with momentum $k$ and energy $\varepsilon_{\mathrm{L}, k}$. The electron tunneling, represented by the Hamiltonian $\hat{H}_{T}$ [37,54], occurs with a strength $t$ and involves the electron operator of the lead $\left[\hat{c}_{\mathrm{L}}^{\dagger}=\int d k \hat{c}_{\mathrm{L}, k}^{\dagger} /(2 \pi)\right]$ and $\operatorname{ABS}\left(\hat{d}_{\mathrm{A}}=u \hat{\gamma}+v \hat{\gamma}^{\dagger}[55]\right)$ where $u \equiv u(x=0)$ and $v \equiv$ $v(x=0)$ are the particle and hole component of the ABS wave function at the junction $(x=0)$. We renormalize the ABS wave function such that $|u|^{2}+|v|^{2}=1$. The ABS-boson coupling term can be derived from the microscopic electronboson interaction by projecting it onto the lowest-energy (ABS) sector (see Sec. I of Ref. [56]). This term can be eliminated using the Lang-Firsov canonical transformation $\hat{\tilde{H}}=e^{\hat{S}} \hat{H} e^{-\hat{S}}$, where $\hat{S}=\frac{\lambda}{\Omega} \hat{\gamma}^{\dagger} \hat{\gamma}\left(\hat{b}^{\dagger}-\hat{b}\right)[53,57]$, which introduces the renormalization $\varepsilon_{\mathrm{A}} \rightarrow \tilde{\varepsilon}_{\mathrm{A}}=\varepsilon_{\mathrm{A}}-\lambda^{2} / \Omega, \hat{b} \rightarrow \hat{\tilde{b}}=$ $\hat{b}-\lambda \hat{\gamma}^{\dagger} \hat{\gamma} / \Omega, \hat{\gamma} \rightarrow \hat{\tilde{\gamma}}=\hat{\gamma} \hat{Y}$ and $\hat{d}_{\mathrm{A}} \rightarrow \hat{\tilde{d}}_{\mathrm{A}}=u \hat{\gamma} \hat{Y}+v \hat{\gamma}^{\dagger} \hat{Y}^{\dagger}$ with $\hat{Y}=e^{-\lambda\left(\hat{b}^{\dagger}-\hat{b}\right) / \Omega}$ (see Sec. II of Ref. [56]). The operator $\hat{Y}$ is analogous to the operator $e^{-i \hat{\varphi}}$ in Ref. [45], through the identification $\lambda\left(\hat{b}^{\dagger}-\hat{b}\right) / \Omega=i \hat{\varphi}$ where $\hat{\varphi}$ is the phase operator of the electromagnetic field used in Ref. [45]. Therefore, our 
results apply generally to all bosonic modes including electromagnetic fields and plasmons.

The current operator is $\hat{I}=-e \dot{\hat{N}}_{\mathrm{L}}=i e\left[\hat{N}_{\mathrm{L}}, \hat{\tilde{H}}_{\mathrm{T}}\right]=$ $i \frac{e}{\hbar}\left(t \hat{c}_{\mathrm{L}}^{\dagger} \hat{\tilde{d}}_{\mathrm{A}}-\right.$ H.c. $)$ where $\dot{\hat{N}}_{\mathrm{L}}$ is the time derivative of the lead electron number. The current is proportional to the tunnel coupling strength $\Gamma \equiv 2 \pi t^{2} v_{0}$ where $v_{0}$ is the density of states at the lead Fermi energy. The ratio $\Gamma / k_{\mathrm{B}} T$ determines two different transport regimes: weak $\left(\Gamma / k_{\mathrm{B}} T<1\right)$ and strong $\left(\Gamma / k_{\mathrm{B}} T>1\right)$ tunneling regimes.

\section{A. Rate equation}

We first study the weak tunneling limit using the rate equation [51,52], which applies for all values of $\lambda$. Without the lead coupling, the eigenstates of the ABS-boson system are $|n, q\rangle$ with eigenenergies $E_{n, q}=n \tilde{\varepsilon}_{\mathrm{A}}+q \Omega$, where the indices $n=0,1$ and $q \in \mathbb{Z}_{\geqslant 0}$ denote the ABS and boson occupation numbers, respectively. The tunneling of electrons and holes from the lead to the ABS introduces transitions between the eigenstates $|n, q\rangle$. If the boson relaxation rate is faster than the tunneling rate $\Gamma / \hbar$ (typically true in experiments [58]) such that the bosons acquire the equilibrium distribution $P_{q}^{\mathrm{b}}=$ $e^{-q \Omega / k_{\mathrm{B}} T}\left(1-e^{-\Omega / k_{\mathrm{B}} T}\right)$, the probability that the system in the state $|n, q\rangle$ can be factorized as $P_{q}^{n}=P^{n} P_{q}^{\mathrm{b}}$. In the steady state, $P^{n}$ satisfies the rate equation (see Sec. III of Ref. [56]):

$$
0=\frac{\partial P^{n}}{\partial t}=P^{\bar{n}} \sum_{p=e, h} R^{\bar{n} \rightarrow n ; p}-P^{n} \sum_{p=e, h} R^{n \rightarrow \bar{n} ; p},
$$

where the probability flux due to the transition from $|\bar{n}\rangle \rightarrow|n\rangle$ and vice versa cancels each other. These transitions rates can be calculated using FGR as (see Sec. III of Ref. [56])

$$
\begin{aligned}
& R^{n \rightarrow \bar{n} ; e}=\frac{\Gamma}{\hbar}\left|\left\langle\bar{n}\left|\hat{d}_{\mathrm{A}}^{\dagger}\right| n\right\rangle\right|^{2} \sum_{q, q^{\prime}} P_{q}^{\mathrm{b}}\left|Y_{q q^{\prime}}\right|^{2} f\left(E_{\bar{n}, q^{\prime}}-E_{n, q}-e V\right), \\
& R^{n \rightarrow \bar{n} ; h}=\frac{\Gamma}{\hbar}\left|\left\langle\bar{n}\left|\hat{d}_{\mathrm{A}}\right| n\right\rangle\right|^{2} \sum_{q, q^{\prime}} P_{q}^{\mathrm{b}}\left|Y_{q q^{\prime}}\right|^{2} f\left(E_{\bar{n}, q^{\prime}}-E_{n, q}+e V\right),
\end{aligned}
$$

where $\left\langle\bar{n}\left|\hat{d}_{\mathrm{A}}^{\dagger}\right| n\right\rangle$ and $\left\langle\bar{n}\left|\hat{d}_{\mathrm{A}}\right| n\right\rangle$ are the bare tunneling matrix elements for electrons and holes, respectively, $Y_{q q^{\prime}}=$ $\left\langle q^{\prime}\left|e^{\lambda\left(\hat{b}^{\dagger}-\hat{b}\right) / \Omega}\right| q\right\rangle$ is the boson emission or absorption matrix element [51] (see Sec. II of Ref. [56]), and $f(E)=[1+$ $\left.\exp \left(E / k_{\mathrm{B}} T\right)\right]^{-1}$ is the lead Fermi function with $T$ being the temperature. Note that $\left|\left\langle 1\left|\hat{d}_{\mathrm{A}}^{\dagger}\right| 0\right\rangle\right|^{2}=\left|\left\langle 0\left|\hat{d}_{\mathrm{A}}\right| 1\right\rangle\right|^{2}=|u|^{2}$ and $\left|\left\langle 0\left|\hat{d}_{\mathrm{A}}^{\dagger}\right| 1\right\rangle\right|^{2}=\left|\left\langle 1\left|\hat{d}_{\mathrm{A}}\right| 0\right\rangle\right|^{2}=|v|^{2}$ (see Sec. II of Ref. [56]). Solving Eq. (3) together with the normalization condition $P^{0}+P^{1}=1$, we obtain $P^{0}$ and $P^{1}$. Substituting these probabilities into the current $I=e \sum_{n} P^{n}\left(R^{n \rightarrow \bar{n} ; e}-R^{n \rightarrow \bar{n} ; h}\right)$ [51], we have

$$
I=2 e \frac{R^{0 \rightarrow 1 ; e} R^{1 \rightarrow 0 ; e}-R^{0 \rightarrow 1 ; h} R^{1 \rightarrow 0 ; h}}{R^{0 \rightarrow 1 ; e}+R^{1 \rightarrow 0 ; h}+R^{1 \rightarrow 0 ; e}+R^{0 \rightarrow 1 ; h}} .
$$

We can show that Eq. (5) reduces to Eq. (1) by noting that the hole tunneling is energetically forbidden at large positive voltages $\left(R^{0 \rightarrow 1 ; h}, R^{1 \rightarrow 0 ; h} \approx 0\right.$ for $\left.e V \gtrsim\left|\tilde{\varepsilon}_{\mathrm{A}}\right|+k_{\mathrm{B}} T\right)$ and so is the electron tunneling at large negative voltages $\left[R^{0 \rightarrow 1 ; e}, R^{1 \rightarrow 0 ; e} \approx 0\right.$ for $\left.e V \lesssim-\left(\left|\tilde{\varepsilon}_{\mathrm{A}}\right|+k_{\mathrm{B}} T\right)\right]$. While Eq. (1) implies PHS for subgap conductances of gapped superconduc-
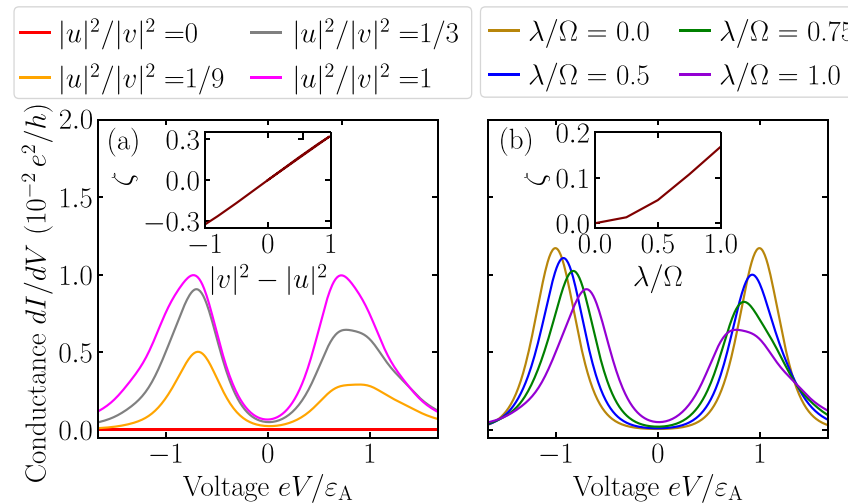

FIG. 2. Conductance $d I / d V$ of boson-coupled ABSs vs voltage $V$ calculated using the rate equation [Eq. (5)]. Conductances for (a) different ratios of $\mathrm{PH}$ components $|u|^{2} /|v|^{2}[59]$ with $\lambda / \Omega=1$ and (b) different ABS-boson coupling strengths $\lambda$ with $|u|^{2} /|v|^{2}=$ $1 / 3$. Inset: (a) Conductance PH asymmetry $\zeta$ vs $|v|^{2}-|u|^{2}$ and (b) $\zeta$ vs $\lambda / \Omega$. Due to the ABS-boson coupling $\lambda$, the ABS energy gets renormalized by $\varepsilon_{\mathrm{A}} \rightarrow \varepsilon_{\mathrm{A}}-\lambda^{2} / \Omega$, which shifts the position of the conductance peaks [panel (b)]. The parameters for all panels are $\varepsilon_{\mathrm{A}} / \Omega=3, \Gamma / \Omega=0.05 /(2 \pi)$, and $k_{\mathrm{B}} T / \Omega=0.4$.

tors, the inclusion of a bosonic bath modifies the tunneling rates in Eq. (1) so as to break the conductance PHS. This PHS breaking can be understood more intuitively in the lowtemperature limit as follows. The first tunneling, occurring with rates $R^{0 \rightarrow 1 ; e}$ [Fig. 1(a)] or $R^{0 \rightarrow 1 ; h}$ [Fig. 1(b)], transfers only lead electrons or holes near the lead Fermi energy and is accompanied by emission of small number of bosons since there are only a few occupied electrons (holes) above (below) the Fermi level. In contrast, the second tunneling, whose rates are $R^{1 \rightarrow 0 ; e}$ [Fig. 1(a)] or $R^{1 \rightarrow 0 ; h}$ [Fig. 1(b)], has a higher probability of boson emission since it transfers electrons and holes with energies deep inside the lead Fermi energy. This means that $R^{0 \rightarrow 1 ; e} \neq R^{1 \rightarrow 0 ; h}$ and $R^{1 \rightarrow 0 ; e} \neq R^{0 \rightarrow 1 ; h}$ for tunneling into ABSs in superconductors with gapless excitations (e.g., phonons) unlike the gapped superconductor case. Therefore, $I\left(V_{0}\right) \neq-I\left(-V_{0}\right)$ [Eq. (1)] and the conductance becomes PH asymmetric, i.e., $\left.\frac{d I}{d V}\right|_{V=V_{0}} \neq\left.\frac{d I}{d V}\right|_{V=-V_{0}}$ (see Sec. IV A of Ref. [56] for a more general proof which holds even for the high-temperature limit).

Figure 2 shows the conductance $d I / d V$ (see Sec. V of Ref. [56] for the current) of boson-coupled ABSs calculated from Eq. (5). As shown in Fig. 2(a), the conductance decreases with increasing ABS's PH content imbalance $\left.|| u\right|^{2}-|v|^{2} \mid$ because the terms $R^{0 \rightarrow 1 ; e} R^{1 \rightarrow 0 ; e}$ and $R^{0 \rightarrow 1 ; h} R^{1 \rightarrow 0 ; h}$ in Eq. (5) are $\propto|u v|^{2}=\left[1-\left(|u|^{2}-|v|^{2}\right)^{2}\right] / 4$. In contrast, the conductance $\mathrm{PH}$ asymmetry magnitude $|\zeta|$ increases linearly with increasing ABS's PH content imbalance $\left.|| u\right|^{2}-|v|^{2} \mid$ [inset of Fig. 2(a)], where

$$
\zeta \equiv \frac{(d I / d V)_{\max ,-}-(d I / d V)_{\max ,+}}{(d I / d V)_{\max ,-}+(d I / d V)_{\max ,+}},
$$

with $(d I / d V)_{\max ,-/+} \equiv \max _{V \leqslant 0 / V \geqslant 0} d I / d V$ being the peak conductance at negative and positive voltages, respectively. $\zeta= \pm 1(\zeta=0)$ corresponds to perfectly asymmetric (symmetric) conductances. Figure 2(b) shows that the peak conductances decrease with increasing ABS-boson coupling 
strength $\lambda$ since $\lambda$ broadens the quasiparticle weight around the ABS energy, which decreases the effective tunnel coupling strength. The conductance PH asymmetry $(\zeta)$ magnitude [60], however, increases with increasing $\lambda$ [inset of Fig. 2(b)] for $\left|\tilde{\varepsilon}_{\mathrm{A}}\right| \gg k_{\mathrm{B}} T$ where the two peaks are well separated. For the regime where $\left|\tilde{\varepsilon}_{\mathrm{A}}\right| \approx k_{\mathrm{B}} T, \zeta$ has a nonmonotonic behavior with $\lambda$ (see Sec. VI of Ref. [56]). Section VI of Ref. [56] shows that $\zeta$ decreases with increasing temperature, depends monotonically on the boson frequency $\Omega$, and prevails only for $\Omega \lesssim 2\left|\tilde{\varepsilon}_{\mathrm{A}}\right|+k_{\mathrm{B}} T$.

\section{B. Keldysh}

For strong-tunneling limit $\left(\Gamma>k_{\mathrm{B}} T\right)$, we compute the current using the mean-field Keldysh formalism. We begin by rewriting Eq. (2a) in terms of the boson displacement $[\hat{x}=$ $\left.\left(\hat{b}+\hat{b}^{\dagger}\right) / \sqrt{2}\right]$ and momentum $\left[\hat{p}=-i\left(\hat{b}-\hat{b}^{\dagger}\right) / \sqrt{2}\right]$ operator as

$$
\hat{H}_{\mathrm{A}}=\varepsilon_{\mathrm{A}} \hat{\gamma}^{\dagger} \hat{\gamma}+\sqrt{2} \lambda \hat{\gamma}^{\dagger} \hat{\gamma} \hat{x}+\frac{\Omega}{2}\left(\hat{x}^{2}+\hat{p}^{2}\right)-\frac{\Omega}{2} .
$$

We calculate the mean-field energy $\varepsilon_{\mathrm{A}}+\sqrt{2} \lambda\langle\hat{x}\rangle$ by selfconsistently solving for $\langle\hat{x}\rangle$ where $\langle\cdots\rangle$ is the expectation value with respect to the mean-field eigenfunction. To this end, we solve for $\partial\left\langle\hat{H}_{\mathrm{A}}\right\rangle / \partial\langle\hat{x}\rangle=0$ and $\partial\left\langle\hat{H}_{\mathrm{A}}\right\rangle / \partial\langle\hat{p}\rangle=0$, giving $\langle\hat{x}\rangle=-\frac{\sqrt{2} \lambda}{\Omega}\left\langle\hat{\gamma}^{\dagger} \hat{\gamma}\right\rangle$ and $\langle\hat{p}\rangle=0$.

The ABS Green's function in the Lehmann representation is $g_{\mathrm{A}}(\omega)=\frac{\Phi_{+} \Phi_{+}^{\dagger}}{\omega-\left(\varepsilon_{\mathrm{A}}+\sqrt{2} \lambda\langle\hat{x}\rangle\right)}+\frac{\Phi_{-} \Phi_{-}^{\dagger}}{\omega+\left(\varepsilon_{\mathrm{A}}+\sqrt{2} \lambda\langle\hat{x}\rangle\right)}$ where $\Phi_{+}=$ $(1,0)^{T}$ and $\Phi_{-}=(0,1)^{T}$ are the Nambu spinors written in the Nambu basis $\left(\hat{\gamma}, \hat{\gamma}^{\dagger}\right)^{T}$. Following Ref. [37], we use the Green's function to evaluate the current as (see Sec. VII of Ref. [56])

$$
I(V)=\frac{e}{h} \Gamma^{2} \int_{-\infty}^{\infty} d \omega \mathcal{A}(\omega)\left[f\left(\omega_{-}\right)-f\left(\omega_{+}\right)\right],
$$

where

$$
\mathcal{A}(\omega)=\frac{4|u v|^{2}}{\left[\omega-\frac{\left(\varepsilon_{\mathrm{A}}+\sqrt{2} \lambda(\hat{x})\right)^{2}}{\omega}-\frac{\left(\Gamma_{u}-\Gamma_{v}\right)^{2}}{4 \omega}\right]^{2}+\left(\Gamma_{u}+\Gamma_{v}\right)^{2}},
$$

with $\Gamma_{u}=\Gamma|u|^{2}$ and $\Gamma_{v}=\Gamma|v|^{2}$. The mean-field boson displacement $\langle\hat{x}\rangle$ in Eq. (9) is evaluated self-consistently as

$$
\langle\hat{x}\rangle=-\frac{\sqrt{2} \lambda}{\Omega}\left\langle\hat{\gamma}^{\dagger} \hat{\gamma}\right\rangle=-\frac{\lambda}{\sqrt{2} \Omega}\left\{1-i \int \frac{d \omega}{2 \pi} \operatorname{Tr}\left[G_{\mathrm{A}}^{<}(\omega) \sigma_{z}\right]\right\},
$$

where $\left(G_{\alpha \beta}^{<}\right)_{\mathrm{A}}=i\left\langle\Psi_{\alpha \mathrm{A}}^{\dagger} \Psi_{\beta \mathrm{A}}\right\rangle$ is the ABS lesser Green's function (see Sec. VIII of Ref. [56]) with $\Psi_{\mathrm{A}}=\left(\hat{\gamma}, \hat{\gamma}^{\dagger}\right)^{T}$ and $\sigma_{z}$ being the $z$-Pauli matrix in the Nambu space.

Figure 3 shows the conductance (see Sec. V of Ref. [56] for the current) of boson-coupled ABSs calculated from Eq. (8) subject to the self-consistency condition [Eq. (10)]. Similar to the rate equation, the conductance of boson-coupled ABSs calculated using the Keldysh approach also decreases with increasing $\mathrm{ABS}$ 's $\mathrm{PH}$ content imbalance $\left.|| u\right|^{2}-|v|^{2} \mid$ [Fig. 3(a)] with its PH asymmetry $(\zeta)$ magnitude increases linearly with increasing $\left.|| u\right|^{2}-|v|^{2} \mid$ [inset of Fig. 3(a)]. Figure 3(b) shows that the peak conductance increases with

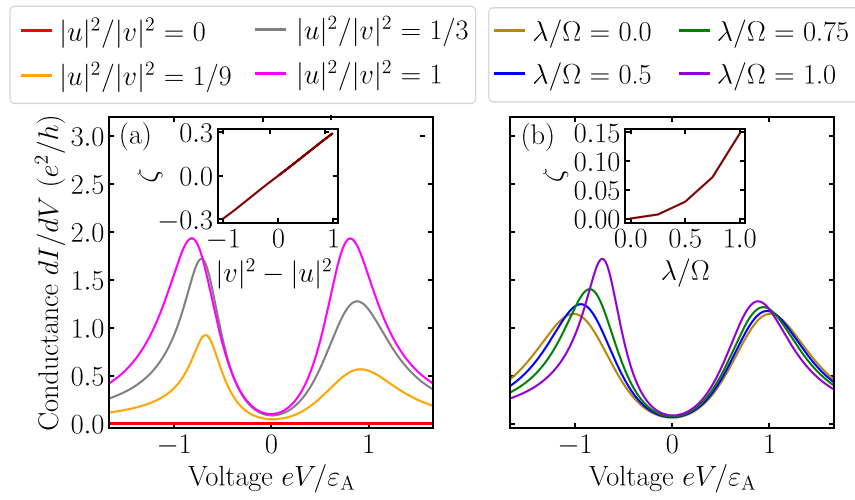

FIG. 3. Conductance $d I / d V$ of boson-coupled ABSs vs voltage $V$ calculated using the mean-field Keldysh approach [Eq. (8)]. Conductances for (a) different ratios of PH components $|u|^{2} /|v|^{2}[59]$ with $\lambda / \Omega=1$ and (b) different ABS-boson coupling strengths $\lambda$ with $|u|^{2} /|v|^{2}=1 / 3$. Inset: (a) Conductance PH asymmetry $\zeta$ vs $|v|^{2}-|u|^{2}$ and (b) $\zeta$ vs $\lambda / \Omega$. Due to the ABS-boson coupling $\lambda$, the ABS energy gets renormalized by $\varepsilon_{\mathrm{A}} \rightarrow \tilde{\varepsilon}_{\mathrm{A}}=\varepsilon_{\mathrm{A}}+\sqrt{2} \lambda\langle\hat{x}\rangle$ (see Sec. V of Ref. [56] for the plots of $\langle\hat{x}\rangle$ ), which shifts the position of the conductance peak [panel (b)]. The parameters for all panels are $\varepsilon_{\mathrm{A}} / \Omega=3, \Gamma / \Omega=2$, and $k_{\mathrm{B}} T / \Omega=0.4$.

increasing ABS-boson coupling strength $\lambda$ contrary to the rate-equation results. However, similar to the rate equation, the conductance $\mathrm{PH}$ asymmetry $\zeta$ increases with increasing $\lambda$ [61]. Unlike the rate equation, the Keldysh approach shows that in the strong-tunneling regime the PHS breaking holds also for high-frequency bosons (see Sec. IX of Ref. [56]), since it arises from nonperturbative effects of tunneling, i.e., the PH asymmetry of the mean-field boson displacement value $\langle\hat{x}\rangle$.

Our model of tunneling into boson-coupled ABS [Eq. (2)] can explain the origin of $\mathrm{PH}$ asymmetry for subgap conductance observed in a hard superconducting gap [5,6,31,32,34], which cannot be accounted for by QP. However, similar to QP this model also results in conductance peak areas which are independent of temperatures (see Sec. IV B of Ref. [56]). In Sec. IV below, we consider another related model, i.e., a boson-assisted tunneling model. This model can not only give rise to PHS breaking of subgap conductances but also account for experimentally observed conductance features which cannot be attributed to QP, e.g., an increase in the conductance peak area with temperature [31].

\section{MODEL II. BOSON-ASSISTED TUNNELING INTO ABS}

In this section, we consider boson-assisted tunneling into an $\mathrm{ABS}$ via virtual hopping of electrons or holes from the lead into higher-lying states in superconductors which are boson-coupled to the ABS. The higher-lying states can be either higher-energy ABSs or states from the continuum above the gap. By integrating out the higher-lying states, we derive the effective low-energy Hamiltonian for the bosonassisted tunneling into the ABS as (see Sec. X of Ref. [56])

$$
\hat{H}_{\mathrm{T}}=t\left(\hat{b}+\hat{b}^{\dagger}\right) \hat{c}_{\mathrm{L}}^{\dagger} \hat{d}_{\mathrm{A}}+\text { H.c. }
$$

Note the extra $\left(\hat{b}+\hat{b}^{\dagger}\right)$ term in the above tunneling Hamiltonian as compared to Eq. (2c) in Sec. III. 

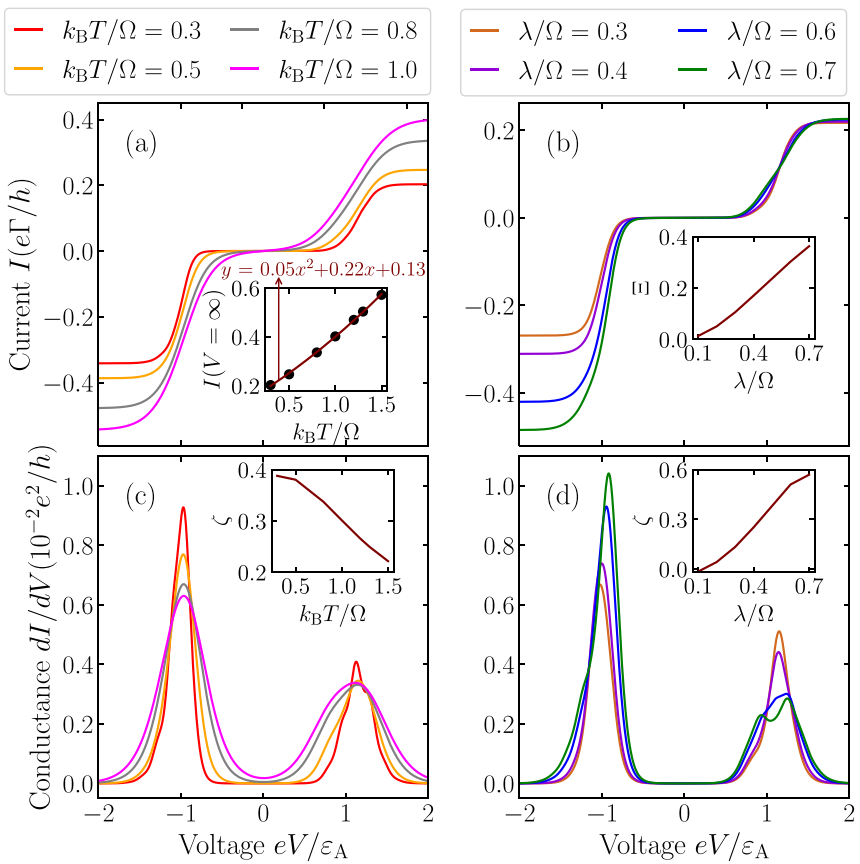

FIG. 4. Current $I$ and conductance $d I / d V$ into ABS vs voltage $V$ for the boson-assisted tunneling model. (Upper panels) Current and (lower panels) conductance for (left panels) different temperatures $k_{\mathrm{B}} T$ with $\lambda / \Omega=0.5$ and (right panels) different ABS-boson coupling strengths $\lambda$ with temperature $k_{\mathrm{B}} T=0.4$. Inset: (a) $I(V=\infty)$ vs $k_{\mathrm{B}} T / \Omega$, (b) Current PH asymmetry $\Xi$ vs $\lambda / \Omega$, (c) Conductance $\mathrm{PH}$ asymmetry $\zeta$ vs $k_{\mathrm{B}} T / \Omega$, and (d) $\zeta$ vs $\lambda / \Omega$. Due to the ABS-boson coupling $\lambda$, the ABS energy gets renormalized by $\varepsilon_{\mathrm{A}} \rightarrow \varepsilon_{\mathrm{A}}-\lambda^{2} / \Omega$, which shifts the position of the conductance peaks [panel (d)]. Note that when $\lambda>k_{\mathrm{B}} T$, e.g., $\lambda / \Omega=0.7$ [see green curve in panel (d)], a second peak (which is the first boson sideband) appears to the right of the ABS peak on the positive-voltage side of the conductance plot; the first boson sideband and the ABS peak are separated by an energy equal to the boson frequency $\Omega$. The parameters for all panels are $|u|=0.1, \varepsilon_{\mathrm{A}} / \Omega=6$, and $\Gamma / \Omega=0.05 /(2 \pi)$.

Figure 4 shows the current and conductance calculated using the rate equation within the boson-assisted tunneling model for different temperatures $T$ and ABS-boson coupling strengths $\lambda$. Contrary to the tunneling model in Sec. III where the current at $V= \pm \infty$ is independent of temperature (see Sec. IV B of Ref. [56]), for the boson-assisted tunneling model the current magnitude at $V= \pm \infty$ increases with increasing temperature. This is because the boson-assisted tunneling rate (see Sec. X of Ref. [56]) is proportional to $\left\langle q\left|\left(\hat{\tilde{b}}+\hat{\tilde{b}}^{\dagger}\right)^{2}\right| q\right\rangle$, which increases with increasing temperature. Crucially, we find that the current $I(V= \pm \infty)$ or equivalently the peak area of the conductance versus voltage curve has a fasterthan-linear increase with temperature [inset of Fig. 4(a)], providing excellent agreement with experimental results [31]. Since QP preserves the conductance peak area under different temperatures and necessarily induces "soft-gap" conductance features, our proposed boson-assisted tunneling process is thus more likely to be responsible for the $\mathrm{PH}$-asymmetric subgap conductances inside a hard superconducting gap observed in Ref. [31].
Contrary to the model in Sec. III, for the boson-assisted tunneling model, the current calculated at large positive and negative voltages need not be perfectly antisymmetric, i.e., $I(V=\infty) \neq-I(V=-\infty)$. The current PH asymmetry $\Xi$, defined as

$$
\Xi \equiv \frac{|I(V=-\infty)|-|I(V=\infty)|}{|I(V=-\infty)|+|I(V=\infty)|},
$$

increases with increasing ABS-boson coupling strength $\lambda$ [see inset of Fig. 4(b)]. This current PH asymmetry (or equivalently the asymmetry between the conductance peak area for positive and negative voltages) as well as the dependence of the conductance peak area with temperature can serve as signatures for the boson-assisted tunneling process. Similar to the model in Sec. III, the conductance PH asymmetry $\zeta$ calculated using the boson-assisted tunneling model also decreases with increasing temperature [inset of Fig. 4(c)] and increases with increasing ABS-boson coupling strength $\lambda$ [inset of Fig. 4(d)].

\section{CONCLUSIONS}

Contrary to widely held belief, we show that the PHS breaking of subgap conductances in superconductors can arise without QP. Specifically, the coupling of ABSs to a bosonic bath (or multimode bosonic baths [62]) can break the PHS of subgap conductances without changing the superconductor's parity state. Therefore, contrary to QP, our mechanism is not detrimental to the coherence of superconductor-based qubits. (Topological qubits are exponentially protected from the bosonic bath dephasing due to the spatial separation of Majoranas [63].) We find that the conductance PH asymmetry increases with increasing ABS's PH content imbalance, increasing ABS-boson coupling strength and decreasing temperature. Our theory is general as it applies to all ABSs, e.g., quasi-Majorana states [64,65], Yu-Shiba-Rusinov states [66-68], Caroli-de Gennes-Matricon states [69], etc., which couple to bosonic modes such as phonons, plasmons, electromagnetic fields, etc., in superconductors. Contrary to QP, our mechanism applies even for ABSs observed inside a hard superconducting gap $[5,6,31,34]$ and can give rise to an increase in the conductance peak area with temperature as observed in experiments [31].

Our PHS breaking mechanism results from boson emissions or absorptions accompanying the electron/hole tunneling. Since these bosons such as phonons are ubiquitous in superconductors, we expect electron-phonon interactions (EPIs) to significantly affect transport in superconductors, particularly the semiconductor-superconductor heterostructures used to realize topological superconductors [3-5,7,8,11,28-30]. In fact, measurements of transport in semiconductors have observed features [70-74] associated with EPI that are theoretically understood [75-77]. We estimate that for a typical topological superconductor which uses either an InAs or InSb semiconductor with a length of $\ell \sim 1 \mu \mathrm{m}$ (having a phonon frequency $\Omega \sim \hbar v_{s} \pi / \ell=7.2 \mu \mathrm{eV}$ where $v_{s} \approx 3.5 \times 10^{5} \mathrm{~cm} / \mathrm{s}$ [78-80] is the sound velocity), EPI can give rise to a 
conductance $\mathrm{PH}$ asymmetry in the tunneling limit for ABSs with energies $\varepsilon_{\mathrm{A}} \gtrsim \Omega / 2=3.6 \mu \mathrm{eV}$. Therefore, contrary to QP, EPI does not affect the zero-bias Majorana conductance.

Compared to diagrammatic techniques, FGR is a more controlled approach in treating the effect of interactions on transport in superconductors (even for strongly correlated superconductors) for the strict tunneling limit. This is because interaction diagrams can generate an imaginary self-energy [37], resulting in a conductance PH asymmetry similar to QP [22]. Therefore, it is crucial to enforce a fermion parity conservation in the diagrammatic treatment of ABS-boson couplings like our mean-field treatment of interactions in the Keldysh formulation. Our work thus motivates the formulation of the nonequilibrium Green's function beyond the mean-field approximation that conserves fermion par- ity. We note that our mechanism is quite distinct from the subgap-conductance PHS breaking due to the bias-voltage dependence of the tunnel barrier [81]. While this mechanism can be treated within the Keldysh approach by moving the interaction term from the ABS to the barrier, it vanishes in the tunneling limit where FGR applies.

\section{ACKNOWLEDGMENTS}

We thank M. Wimmer, J. Saldana, and R. Hanai for useful discussions. This work was supported by Microsoft research, Army Research Office Grants No. W911NF-19-1-0328, NSF DMR-1555135 (CAREER), JQI-NSF-PFC (supported by NSF grant PHY-1607611), and NSF PHY-1748958 (through helpful discussions at KITP).
[1] V. Mourik, K. Zuo, S. M. Frolov, S. R. Plissard, E. P. A. M. Bakkers, and L. P. Kouwenhoven, Signatures of Majorana fermions in hybrid superconductor-semiconductor nanowire devices, Science 336, 1003 (2012).

[2] S. Nadj-Perge, I. K Drozdov, J. Li, H. Chen, S. Jeon, J. Seo, A. H. MacDonald, B. A. Bernevig, and A. Yazdani, Observation of Majorana fermions in ferromagnetic atomic chains on a superconductor, Science 346, 602 (2014).

[3] H. J. Suominen, M. Kjaergaard, A. R. Hamilton, J. Shabani, C. J. Palmstrøm, C. M. Marcus, and F. Nichele, Zero-Energy Modes from Coalescing Andreev States in a Two-Dimensional Semiconductor-Superconductor Hybrid Platform, Phys. Rev. Lett. 119, 176805 (2017).

[4] F. Nichele, A. C. C. Drachmann, A. M. Whiticar, E. C. T. O'Farrell, H. J. Suominen, A. Fornieri, T. Wang, G. C. Gardner, C. Thomas, A. T. Hatke et al., Scaling of Majorana Zero-Bias Conductance Peaks, Phys. Rev. Lett. 119, 136803 (2017).

[5] G. C. Ménard, S. Guissart, C. Brun, R. T. Leriche, M. Trif, F. Debontridder, D. Demaille, D. Roditchev, P. Simon, and T. Cren, Two-dimensional topological superconductivity in $\mathrm{Pb} / \mathrm{Co} / \mathrm{Si}(111)$, Nat. Commun. 8, 2040 (2017).

[6] D.-J. Choi, C. Rubio-Verdú, J. de Bruijckere, M. M. Ugeda, N. Lorente, and J. I. Pascual, Mapping the orbital structure of impurity bound states in a superconductor, Nat. Commun. 8, 15175 (2017).

[7] Ö. Gül, H. Zhang, J. D. S. Bommer, M. W. A. de Moor, D. Car, S. R. Plissard, E. P. A. M. Bakkers, A. Geresdi, K. Watanabe, T. Taniguchi et al., Ballistic Majorana nanowire devices, Nat. Nanotechnol. 13, 192 (2018).

[8] M.-T. Deng, S. Vaitiekenas, E. Prada, P. San-Jose, J. Nygård, P. Krogstrup, R. Aguado, and C. M. Marcus, Nonlocality of majorana modes in hybrid nanowires, Phys. Rev. B 98, 085125 (2018).

[9] A. Fornieri, A. M. Whiticar, F. Setiawan, E. Portolés, A. C. C. Drachmann, A. Keselman, S. Gronin, C. Thomas, T. Wang, R. Kallaher et al., Evidence of topological superconductivity in planar Josephson junctions, Nature (London) 569, 89 (2019).

[10] H. Ren, F. Pientka, S. Hart, A. T. Pierce, M. Kosowsky, L. Lunczer, R. Schlereth, B. Scharf, E. M. Hankiewicz, L. W. Molenkamp et al., Topological superconductivity in a phasecontrolled Josephson junction, Nature (London) 569, 93 (2019).
[11] S. Vaitiekenas, G. W. Winkler, B. van Heck, T. Karzig, M.-T. Deng, K. Flensberg, L. I. Glazman, C. Nayak, P. Krogstrup, R. M. Lutchyn et al., Flux-induced topological superconductivity in full-shell nanowires, Science 367, 6485 (2020).

[12] Z. Wang, J. O. Rodriguez, L. Jiao, S. Howard, M. Graham, G. D. $\mathrm{Gu}$, T. L. Hughes, D. K. Morr, and V. Madhavan, Evidence for dispersing 1D Majorana channels in an iron-based superconductor, Science 367, 104 (2020).

[13] H. Zhang, M. W. A. de Moor, J. D. S. Bommer, D. Xu, G. Wang, N. van Loo, C.-X. Liu, S. Gazibegovic, J. A. Logan, D. Car et al., Large zero-bias peaks in InSb-Al hybrid semiconductorsuperconductor nanowire devices, arXiv:2101.11456 (2021).

[14] A. Y. Kitaev, Fault-tolerant quantum computation by anyons, Ann. Phys. 303, 2 (2003).

[15] C. Nayak, S. H. Simon, A. Stern, M. Freedman, and S. Das Sarma, Non-Abelian anyons and topological quantum computation, Rev. Mod. Phys. 80, 1083 (2008).

[16] K. T. Law, P. A. Lee, and T. K. Ng, Majorana Fermion Induced Resonant Andreev Reflection, Phys. Rev. Lett. 103, 237001 (2009).

[17] K. Flensberg, Tunneling characteristics of a chain of Majorana bound states, Phys. Rev. B 82, 180516(R) (2010).

[18] M. Wimmer, A. R. Akhmerov, J. P. Dahlhaus, and C. W. J. Beenakker, Quantum point contact as a probe of a topological superconductor, New J. Phys. 13, 053016 (2011).

[19] F. Setiawan, P. M. R. Brydon, J. D. Sau, and S. Das Sarma, Conductance spectroscopy of topological superconductor wire junctions, Phys. Rev. B 91, 214513 (2015).

[20] G. E. Blonder, M. Tinkham, and T. M. Klapwijk, Transition from metallic to tunneling regimes in superconducting microconstrictions: Excess current, charge imbalance, and supercurrent conversion, Phys. Rev. B 25, 4515 (1982).

[21] G. B. Lesovik, A. L. Fauchère, and G. Blatter, Nonlinearity in normal-metal-superconductor transport: Scattering-matrix approach, Phys. Rev. B 55, 3146 (1997).

[22] I. Martin and D. Mozyrsky, Nonequilibrium theory of tunneling into a localized state in a superconductor, Phys. Rev. B 90, 100508(R) (2014).

[23] A. Yazdani, B. A. Jones, C. P. Lutz, M. F. Crommie, and D. M. Eigler, Probing the local effects of magnetic impurities on superconductivity, Science 275, 1767 (1997). 
[24] K. Matsuba, H. Sakata, N. Kosugi, H. Nishimori, and N. Nishida, Ordered vortex lattice and intrinsic vortex core states in $\mathrm{Bi}_{2} \mathrm{Sr}_{2} \mathrm{CaCu}_{2} \mathrm{O}_{x}$ studied by scanning tunneling microscopy and spectroscopy, J. Phys. Soc. Jpn. 72, 2153 (2003).

[25] L. Shan, Y.-L. Wang, B. Shen, B. Zeng, Y. Huang, A. Li, D. Wang, H. Yang, C. Ren, Q.-H. Wang et al., Observation of ordered vortices with Andreev bound states in $\mathrm{Ba}_{0.6} \mathrm{~K}_{0.4} \mathrm{Fe}_{2} \mathrm{As}_{2}$, Nat. Phys. 7, 325 (2011).

[26] T. Hanaguri, K. Kitagawa, K. Matsubayashi, Y. Mazaki, Y. Uwatoko, and H. Takagi, Scanning tunneling microscopy/spectroscopy of vortices in LiFeAs, Phys. Rev. B 85, 214505 (2012).

[27] M. Chen, X. Chen, H. Yang, Z. Du, X. Zhu, E. Wang, and H.$\mathrm{H}$. Wen, Discrete energy levels of Caroli-De Gennes-Matricon states in quantum limit in $\mathrm{FeTe}_{0.55} \mathrm{Se}_{0.45}$, Nat. Commun. 9, 970 (2018).

[28] J. D. S. Bommer, H. Zhang, Ö. Gül, B. Nijholt, M. Wimmer, F. N. Rybakov, J. Garaud, D. Rodic, E. Babaev, M. Troyer et al., Spin-Orbit Protection of Induced Superconductivity in Majorana Nanowires, Phys. Rev. Lett. 122, 187702 (2019).

[29] J. Chen, B. D. Woods, P. Yu, M. Hocevar, D. Car, S. R. Plissard, E. P. A. M. Bakkers, T. D. Stanescu, and S. M. Frolov, Ubiquitous Non-Majorana Zero-Bias Conductance Peaks in Nanowire Devices, Phys. Rev. Lett. 123, 107703 (2019).

[30] P. Yu, J. Chen, M. Gomanko, G. Badawy, E. P. A. M. Bakkers, K. Zuo, V. Mourik, and S. M. Frolov, Non-Majorana states yield nearly quantized conductance in proximatized nanowires, Nat. Phys. 17, 482 (2021).

[31] J. C. E. Saldaña, A. Vekris, V. Sosnovtseva, T. Kanne, P. Krogstrup, K. Grove-Rasmussen, and J. Nygård, Temperature induced shifts of Yu-Shiba-Rusinov resonances in nanowirebased hybrid quantum dots, Commun. Phys. 3, 125 (2020).

[32] L. Farinacci, G. Ahmadi, M. Ruby, G. Reecht, B. W. Heinrich, C. Czekelius, F. von Oppen, and K. J. Franke, Interfering Tunneling Paths Through Magnetic Molecules on Superconductors: Asymmetries of Kondo and Yu-Shiba-Rusinov Resonances, Phys. Rev. Lett. 125, 256805 (2020).

[33] D. Wang, J. Wiebe, R. Zhong, G. Gu, and R. Wiesendanger, Spin-Polarized Yu-Shiba-Rusinov States in an Iron-Based Superconductor, Phys. Rev. Lett. 126, 076802 (2021).

[34] H. Ding, Y. Hu, M. T. Randeria, S. Hoffman, O. Deb, J. Klinovaja, D. Loss, and A. Yazdani, Tuning interactions between spins in a superconductor, Proc. Natl. Acad. Sci. USA 118, e2024837118 (2021).

[35] S. Das Sarma, A. Nag, and J. D. Sau, How to infer non-Abelian statistics and topological visibility from tunneling conductance properties of realistic Majorana nanowires, Phys. Rev. B 94, 035143 (2016).

[36] C.-X. Liu, J. D. Sau, and S. Das Sarma, Role of dissipation in realistic Majorana nanowires, Phys. Rev. B 95, 054502 (2017).

[37] M. Ruby, F. Pientka, Y. Peng, F. von Oppen, B. W. Heinrich, and K. J. Franke, Tunneling Processes into Localized Subgap States in Superconductors, Phys. Rev. Lett. 115, 087001 (2015).

[38] J. Aumentado, M. W. Keller, J. M. Martinis, and M. H. Devoret, Nonequilibrium Quasiparticles and $2 e$ Periodicity in SingleCooper-Pair Transistors, Phys. Rev. Lett. 92, 066802 (2004).

[39] A. P. Higginbotham, S. M. Albrecht, G. Kiršanskas, W. Chang, F. Kuemmeth, P. Krogstrup, T. S. Jespersen, J. Nygård, K. Flensberg, and C. M. Marcus, Parity lifetime of bound states in a proximitized semiconductor nanowire, Nat. Phys. 11, 1017 (2015).

[40] S. M. Albrecht, E. B. Hansen, A. P. Higginbotham, F. Kuemmeth, T. S. Jespersen, J. Nygård, P. Krogstrup, J. Danon, K. Flensberg, and C. M. Marcus, Transport Signatures of Quasiparticle Poisoning in a Majorana Island, Phys. Rev. Lett. 118, 137701 (2017).

[41] G. Goldstein and C. Chamon, Decay rates for topological memories encoded with Majorana fermions, Phys. Rev. B 84, 205109 (2011).

[42] D. Rainis and D. Loss, Majorana qubit decoherence by quasiparticle poisoning, Phys. Rev. B 85, 174533 (2012).

[43] J. C. Budich, S. Walter, and B. Trauzettel, Failure of protection of Majorana based qubits against decoherence, Phys. Rev. B 85 , 121405(R) (2012).

[44] A. O. Caldeira and A. J. Leggett, Quantum tunnelling in a dissipative system, Ann. Phys. 149, 374 (1983).

[45] G.-L. Ingold and Yu. V. Nazarov, Charge tunneling rates in ultrasmall junctions, in Single Charge Tunneling, edited by $\mathrm{H}$. Grabert and M. H. Devoret, NATO ASI Series B, Vol. 294 (Springer, Boston, MA, 1992), pp. 21-107.

[46] S. M. Shapiro, G. Shirane, and J. D. Axe, Measurements of the electron-phonon interaction in nb by inelastic neutron scattering, Phys. Rev. B 12, 4899 (1975).

[47] B. Friedl, C. Thomsen, and M. Cardona, Determination of the Superconducting Gap in $R \mathrm{Ba}_{2} \mathrm{Cu}_{3} \mathrm{O}_{7-\delta}$, Phys. Rev. Lett. 65, 915 (1990).

[48] M. Hepting, L. Chaix, E. W. Huang, R. Fumagalli, Y. Y. Peng, B. Moritz, K. Kummer, N. B. Brookes, W. C. Lee, M. Hashimoto et al., Three-dimensional collective charge excitations in electron-doped copper oxide superconductors, Nature (London) 563, 374 (2018).

[49] J. Majer, J. M. Chow, J. M. Gambetta, J. Koch, B. R. Johnson, J. A. Schreier, L. Frunzio, D. I. Schuster, A. A. Houck, A. Wallraff et al., Coupling superconducting qubits via a cavity bus, Nature (London) 449, 443 (2007).

[50] A. J. Leggett, S. Chakravarty, A. T. Dorsey, M. P. A. Fisher, A. Garg, and W. Zwerger, Dynamics of the dissipative two-state system, Rev. Mod. Phys. 59, 1 (1987).

[51] A. Mitra, I. Aleiner, and A. J. Millis, Phonon effects in molecular transistors: Quantal and classical treatment, Phys. Rev. B 69, 245302 (2004)

[52] J. Koch, F. von Oppen, Y. Oreg, and E. Sela, Thermopower of single-molecule devices, Phys. Rev. B 70, 195107 (2004).

[53] G. D. Mahan, Many-Particle Physics, 3rd ed. (Kluwer Academic/Plenum Publishers, New York, 2000).

[54] A. V. Balatsky, I. Vekhter, and J.-X. Zhu, Impurity-induced states in conventional and unconventional superconductors, Rev. Mod. Phys. 78, 373 (2006).

[55] Since we consider only the subgap state and ignore the abovegap states, the relation $\hat{d}_{\mathrm{A}}=u \gamma+v \gamma^{\dagger}$ is only approximate, which makes $\hat{d}_{\mathrm{A}}$ nonfermionic. The operator $\hat{d}_{\mathrm{A}}$ becomes fermionic if all the states in the superconductor including the above-gap states are taken into account [see Eq. (S-5) in Sec. I of Ref. [56]]. Our conclusion on the PHS breaking of the subgap conductance due to the ABS-boson coupling does not rely on the fermionic properties of $\hat{d}_{\mathrm{A}}$.

[56] See Supplemental Material at http://link.aps.org/supplemental/ 10.1103/PhysRevResearch.3.L032038 for (I) derivation of the ABS-boson coupling term from the microscopic electron-boson 
interaction, (II) Lang-Firsov transformation, (III) derivations of the rate equation and FGR tunneling rates, (IV) proof for the particle-hole asymmetry of boson-coupled-ABS conductance and proof for the temperature independence of the conductance peak area for the boson-coupled ABS model (model I), (V) current calculated from the rate equation and Keldysh approach, (VI) dependence of the current and conductance calculated from the rate equation on the ABS-boson coupling strength, temperature, boson frequency, and ABS energy, (VII) derivation of the current in the Keldysh formalism, (VIII) explicit expressions for $G_{\mathrm{A}}^{<,>}(\omega)$, (IX) dependence of the current and conductance calculated from Keldysh approach on the ABSboson coupling strength, temperature, boson frequency and lead-tunnel coupling strength, and (X) details on model II (boson-assisted tunneling into ABS). The Supplemental Material includes Refs. [37,51-54,57,82-86].

[57] I. G. Lang and Y. A. Firsov, Kinetic theory of semiconductors with low mobility, Sov. Phys. JETP 16, 1301 (1963).

[58] V. F. Maisi, Andreev tunneling and quasiparticle excitations in mesoscopic normal metal-superconductor structures, Ph.D. thesis, Aalto University, Helsinki, Finland, 2014, http://www. vtt.fi/inf/pdf/MIKES/2014-J1.pdf.

[59] The conductance for $|v|^{2}>|u|^{2}$ can be obtained from the conductance for $|v|^{2}<|u|^{2}$ (shown in Figs. 2 and 3) by interchanging both $|u| \leftrightarrow|v|$ and $V \leftrightarrow-V$ simultaneously. As a result, the higher and lower peaks switch sides which changes the sign of the PH asymmetry $\zeta$ (see Sec. IV A of Ref. [56] for a proof).

[60] The increase of the conductance $\mathrm{PH}$ asymmetry with increasing ABS's PH content imbalance or ABS-phonon coupling strength also holds for the boson sidebands. Here we focus on the regime $k_{\mathrm{B}} T \gtrsim \lambda$ where the boson sidebands vanish due to the thermal broadening [51].

[61] Since we ignore the Fock term in the mean-field approximation, the conductance calculated from the Keldysh approach has no boson sidebands.

[62] While here we focus exclusively on single-mode bosonic baths, our proposed mechanism is expected to hold also for multimode bosonic baths since the result for multimode bosonic baths is qualitatively similar to averaging multiple results for different single-bosonic modes. This averaging is justified as bosonic modes do not interact with each other and are therefore independent.

[63] C. Knapp, T. Karzig, R. M. Lutchyn, and C. Nayak, Dephasing of Majorana-based qubits, Phys. Rev. B 97, 125404 (2018).

[64] G. Kells, D. Meidan, and P. W. Brouwer, Near-zero-energy end states in topologically trivial spin-orbit coupled superconducting nanowires with a smooth confinement, Phys. Rev. B 86, 100503(R) (2012).

[65] C.-X. Liu, J. D. Sau, T. D. Stanescu, and S. Das Sarma, Andreev bound states versus Majorana bound states in quantum dot-nanowire-superconductor hybrid structures: Trivial versus topological zero-bias conductance peaks, Phys. Rev. B 96, 075161 (2017).

[66] L. Yu, Bound state in superconductors with paramagnetic impurities, Acta Phys. Sin. 21, 75 (1965).

[67] H. Shiba, Classical spins in superconductors, Prog. Theor. Phys. 40, 435 (1968).
[68] A. I. Rusinov, Superconductivity near paramagnetic impurities, JETP Lett. 9, 85 (1969).

[69] C. Caroli, P. G. De Gennes, and J. Matricon, Bound Fermion States on a Vortex Line in a Type II Superconductor, Phys. Lett. 9, 307 (1964).

[70] J. R. Huntzinger, J. Groenen, M. Cazayous, A. Mlayah, N. Bertru, C. Paranthoen, O. Dehaese, H. Carrère, E. Bedel, and G. Armelles, Acoustic-phonon Raman scattering in InAs/InP self-assembled quantum dots, Phys. Rev. B 61, R10547 (2000).

[71] V. J. Goldman, D. C. Tsui, and J. E. Cunningham, Evidence for LO-phonon-emission-assisted tunneling in double-barrier heterostructures, Phys. Rev. B 36, 7635 (1987).

[72] T. R. Hartke, Y.-Y. Liu, M. J. Gullans, and J. R. Petta, Microwave Detection of Electron-Phonon Interactions in a Cavity-Coupled Double Quantum Dot, Phys. Rev. Lett. 120, 097701 (2018).

[73] P. Roulleau, S. Baer, T. Choi, F. Molitor, J. Güttinger, T. Müller, S. Dröscher, K. Ensslin, and T. Ihn, Coherent electron-phonon coupling in tailored quantum systems, Nat. Commun. 2, 239 (2011).

[74] C. Weber, A. Fuhrer, C. Fasth, G. Lindwall, L. Samuelson, and A. Wacker, Probing Confined Phonon Modes by Transport Through a Nanowire Double Quantum Dot, Phys. Rev. Lett. 104, 036801 (2010).

[75] E. A. Muljarov and R. Zimmermann, Dephasing in Quantum Dots: Quadratic Coupling to Acoustic Phonons, Phys. Rev. Lett. 93, 237401 (2004).

[76] L. Kleinman, Theory of phonon-assisted tunneling in semiconductors, Phys. Rev. 140, A637 (1965).

[77] N. S. Wingreen, K. W. Jacobsen, and J. W. Wilkins, Resonant Tunneling With Electron-Phonon Interaction: An Exactly Solvable Model, Phys. Rev. Lett. 61, 1396 (1988).

[78] M. Yano, H. Furuse, Y. Iwai, K. Yoh, and M. Inoue, Raman scattering analysis of InAs/GaSb ultrathin-layer superlattices grown by molecular beam epitaxy, J. Cryst. Growth 127, 807 (1993).

[79] J. Wagner, J. Schmitz, N. Herres, J. D. Ralston, and P. Koidl, Raman scattering by folded longitudinal acoustic phonons in InAs/GaSb superlattices: Resonant enhancement and effect of interfacial bonding, Appl. Phys. Lett. 66, 3498 (1995).

[80] O. Madelung, Semiconductors: Group IV Elements and III-V Compounds (Springer Science \& Business Media, 2012).

[81] A. Melo, C.-X. Liu, P. Rożek, T. O. Rosdahl, and M. Wimmer, Conductance asymmetries in mesoscopic superconducting devices due to finite bias, SciPost Phys. 10, 037 (2021).

[82] J. C. Cuevas, A. Martín-Rodero, and A. L. Yeyati, Hamiltonian approach to the transport properties of superconducting quantum point contacts, Phys. Rev. B 54, 7366 (1996).

[83] S. A. González, L. Melischek, O. Peters, K. Flensberg, K. J. Franke, and F. von Oppen, Photon-assisted resonant Andreev reflections: Yu-Shiba-Rusinov and Majorana states, Phys. Rev. B 102, 045413 (2020).

[84] D. Rogovin and D. J. Scalapino, Fluctuation phenomena in tunnel junctions, Ann. Phys. 86, 1 (1974).

[85] H. Haug and A.-P. Jauho, Quantum Kinetics in Transport and Optics of Semiconductors (Springer, 2008), Vol. 2.

[86] A. Kamenev and A. Levchenko, Keldysh technique and nonlinear $\sigma$-model: Basic principles and applications, Adv. Phys. 58, 197 (2010). 